\title{
"Epic Ear Defence"-A Game to Educate Children on the Risks of Noise-Related Hearing Loss
}

\author{
Robert H. Eikelboom, BE, MAppISc, PhD, ${ }^{1-3}$ Natalie F. Leishman, BSc, ${ }^{1,2}$ Tyler J. Munro, BSc, ${ }^{1,2,4}$ \\ Bach Nguyen, BSc, ${ }^{2,4}$ Peter R. Riggs, BSc, ${ }^{1,2,4}$ Jonathon Tennant, BSc, ${ }^{2,4}$ \\ Rhiannon K. West, BSc, ${ }^{1,2}$ and William B. Robertson, BSc, MSc, PhD ${ }^{1,2}$
}

\begin{abstract}
Hearing loss resulting from overexposure to entertainment-related sounds is a modern concern. "Epic Ear Defence" places the player in the three-dimensional environment of the ear canal and challenges the player to defend the ear from various noises, to delay the onset of noise-related hearing loss.
\end{abstract}

\section{Background}

$\mathbf{H}$ EARING LOSS IS ONE of the most common disabilities in the world. ${ }^{1}$ A major contributor to hearing loss is noise exposure. ${ }^{2}$ Exposure to high levels of sound for prolonged periods causes permanent damage to the hair cells in the cochlea.

After World War II much attention was paid to war-related and subsequently occupational-related noise exposure, resulting in regulatory and legislative measures being put in place in many countries to minimize risks. These include limiting time of exposure relating to the loudness of the noise, use of hearing protection, and reducing noise at the source.

Hearing loss is a significant cost to the community, affecting one in six people, and costing over $\$ 11$ billion per annum to the Australian economy. ${ }^{3}$ Hearing loss is particularly significant for children as it has a profound influence on their development and learning, limiting education achievement and future employment.

In recent decades the awareness of the risks of recreational noise has increased, with several studies linking prolonged exposure to noise to hearing loss in children ${ }^{4}$ and teenagers. ${ }^{5}$ These risks have increased significantly with the widespread use of personal listening devices. These devices can produce sound levels up to $106 \mathrm{~dB}$; the recommended daily exposure time at this level is 3.75 minutes.

As children and teenagers are large consumers of music using personal listening devices, it is important that they are educated on the risks of permanent hearing loss and given advice and strategies on how to minimize the risks.

We have developed and implemented a classroom-based health promotion program focusing on noise induced hearing loss. Called "Cheers for Ears" it has been delivered to over 22,000 10-12 year olds since its launch in 2010. Each session lasts for 1 hour and utilizes demonstrations and interactive exercises with the children delivered by a health promotion officer. Other program innovations include a Web site, a manikin-based hearing loss simulator, a noise dosimeter for Android $^{\circledR}$ (Google) devices called Safe\&Sound, and a Mascot dubbed "Cheers for Ears Charlie."

To enable the "Cheers for Ears" messages to reach beyond classroom sessions, the idea of a computer game was conceived. Reinforcement of the messages by follow-up sessions or other means has been shown to be effective. Furthermore, health promotion programs, such as "Cheers for Ears," will always be restricted by limitations on human resources, and their reach is often limited to a local area, with rural and remotely located people often unable to access these programs.

A four-person team of final-year Games Technology students was given a design brief to develop an educational computer game that included the "Cheers for Ears" messages that hearing loss is permanent, that it affected enjoyment of listening to music, and that lowering the volume of sounds is an effective way of minimizing the risk of hearing loss. It provided gameplay elements that helped players gain an intuitive understanding of how the human auditory system works and how excessive noise can permanently damage hearing.

\section{Design Brief and Approach}

Consideration in the development and implementation of the project were as follows:

1. The game should be engaging for a young audience, especially 10-12 year olds.

\footnotetext{
${ }^{1}$ Ear Sciences Centre, School of Surgery, The University of Western Australia, Nedlands, Western Australia, Australia.

${ }^{2}$ Ear Science Institute Australia, Subiaco, Western Australia, Australia.

${ }^{3}$ Department of Communication Pathology, University of Pretoria, Pretoria, South Africa.

${ }^{4}$ School of Information Technology, Murdoch University, Murdoch, Western Australia, Australia.
} 
2. It should be subtle in delivering the messages and not include violence.

3. To achieve as wide an audience as possible, it should be available at least on personal computers with an option to play it on mobile devices as well.

The design team decided to use an incremental development methodology. This approach allowed for each piece of the development to be completed and tested before proceeding to the next increment. It also allowed for the design brief to be modified during the development process.

It was decided to develop the game in a three-dimensional space, set in the ear canal, with the player controlling the actions of character on the screen and with the game coming to a conclusion. In line with a requirement that we did not give the impression that listening to music was undesirable, the game was not to destroy or eliminate the "enemies" but to reduce their effect.

The Unity 3D Game Engine (unity3d.com) was chosen as a development engine because it provided ready-made modules to assemble a three-dimensional game that could be published on multiple platforms. C\# was the programming language. The iTween animation library from unity was utilized. Three-dimensional models were created with Autodesk $^{\circledR}$ (San Rafael, CA) 3ds Max ${ }^{\circledR}$ and ported into the game engine.

\section{Description of the Game}

"Epic Ear Defence" is set in the human ear canal, normally about $20 \mathrm{~mm}$ long and $10 \mathrm{~mm}$ in diameter. The face of an earphone is simulated at the entrance to the ear canal, and the ear drum is the limit of the playing area at the other end. Undulations in the ear canal limit the extent of the view to the player. Ear canal hairs are simulated as obstructions to moving about the canal. The action takes place on the floor of the canal. The aim of the game is to reduce the "volume" of "sound enemies" before they reach the ear drum. The game's genre can be described as a mixture of "third person over-theshoulder action" and "tower defense."

There are three "enemy" characters: "Fearbuds," in the shape of an earphone bud with an audio connector as a tail and representing mid-frequency sounds (Fig. 1); "tweeters," in the shape of a sports whistle and representing high-frequency sounds; and "bass kickers," in the shape of a speaker box and representing low-frequency sounds. Each of the enemy types trails a sine wave representing sound energy, which changes in height and from red to green in color as the volume of the enemy is decreased and then to a white color when their energy is completely depleted. The enemies originate randomly and in increasing frequency from the earphone bud.

The ear is defended by the game's main character, who has the options of using a ray gun to sap energy from the enemies

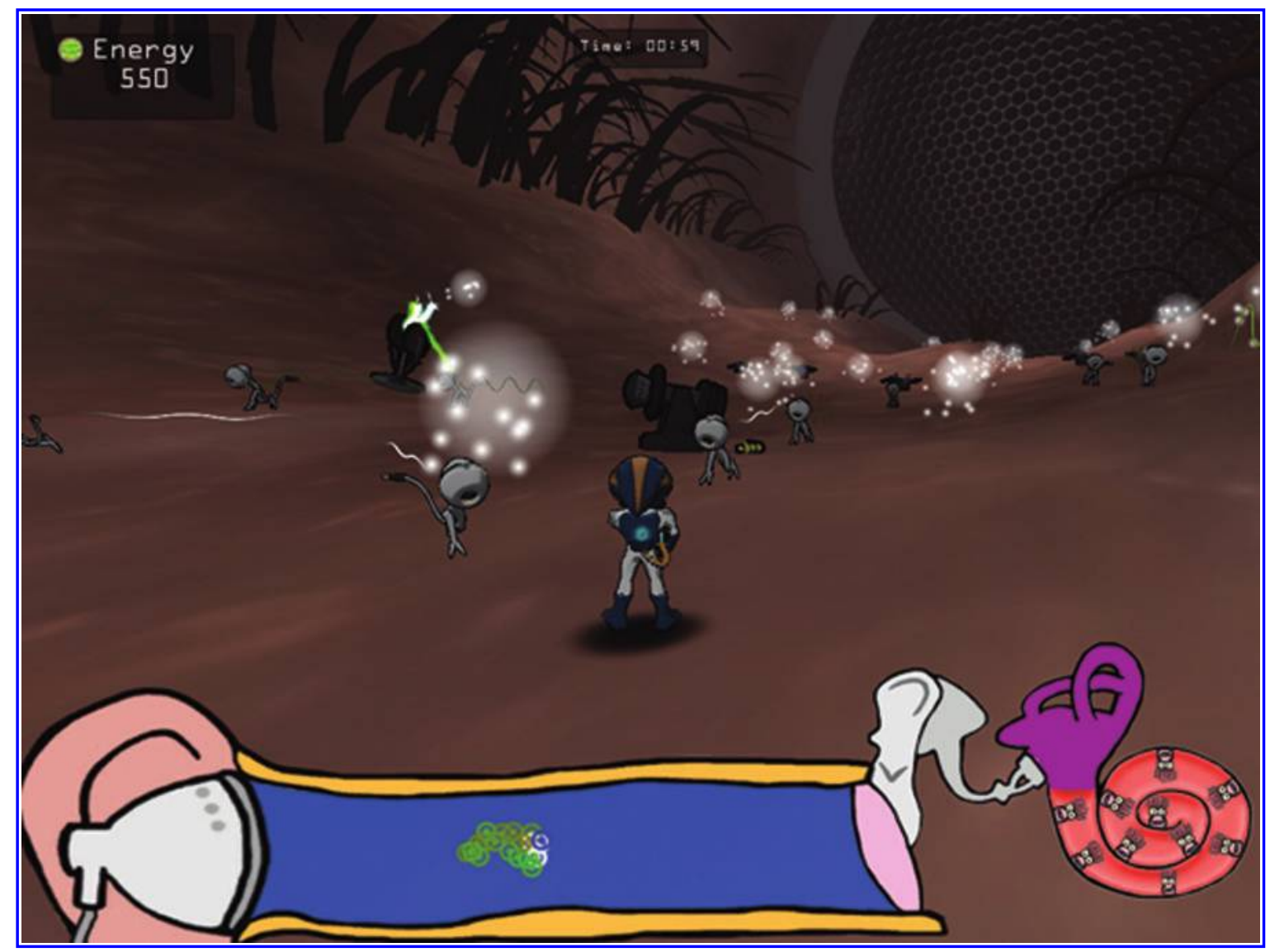

FIG. 1. "Epic Ear Defence" soon after the start of the game. The shield level is high (all blue in the heads-up display), and all 10 hair cell elements are intact (lower right). The game character is being approached by a wave of earphone buds. The one closest to the game character has already had its energy lowered, as seen by the white trail. The white glowing orbs are locations where the player can choose to build a defensive tower. In the distance, a sapper tower can be seen on the left, and an earplug cannon can be seen on the right. Color images available online at www.liebertonline.com/g4h 
or building defensive "towers" in strategic positions. The game character is moved by the conventional "a-s-d-w" combination of keys. Aiming and using the ray gun are done by moving the mouse and clicking the left mouse button, respectively.

The player is assisted by three defensive units: Ear wax gland to slow the enemies, sapper towers to quickly sap a small amount of energy of passing enemies, and a plug cannon, a slow firing way to reduce larger amounts of energy (Fig. 2). They can be placed at strategic locations when the player has gained sufficient credit to "buy" them, and both the sapper towers and cannons can be upgraded by two levels using more of the available credit to become more effective. Credit (the game currency, shown as player energy) is gained by sapping the energy from the enemies.

Eventually all enemies will reach the eardrum. As they fire off their remaining energy against the eardrum, they affect the rest of the hearing system. Damage to the hair cells is dose-related, a product of time and energy. The defensive ability of the hearing system is represented as a shield status. When this reaches zero, one of the 10 hair cell elements is eliminated. When there is no attack on the eardrum, the shield status recovers. Over time the accumulation of energy results in the loss of all the hairs cells, and the game ends. The goal of the player is to last as long as possible and gain a high score. Points are added to the player's score as he or she effectively reduces the volume of the sound enemies.

The status of the hearing is represented in a number of ways. Placed at the bottom of the screen is the heads-up display, which is a stylized two-dimensional inner ear diagram that shows the whole of the play area, the location of the game character, enemies, and defensive units, the status of shield as a moving bar, and the surviving and eliminated hair cells. A background sound track is modified as hair cells are lost to represent the effect of hearing loss on music. The player's energy level and score are shown numerically on the screen.

\section{Testing and Implementation}

A tutorial for "Epic Ear Defence" was developed to introduce novices to controlling the game character and the various game elements. A "trailer" was also written for promotional purposes. The game was tested with a group of Year 7 students to gauge the balance of the game (i.e., too difficult or too simple). As a result the balance was adjusted to make it slightly more difficult. It was also noted that the children quickly understood how to play the game and that they grasped the underlying ear health messages.

Windows and iOS operating system versions were created and made available for downloading and playing on the "Cheers for Ears" Web site.

\section{Conclusions and Further Development}

The task of delivering a serious health message through the medium of a computer game was considered a challenge for all parties at the start of the project. However, we were able to demonstrate that the combination of skills of programmers and hearing health educators were able to successfully

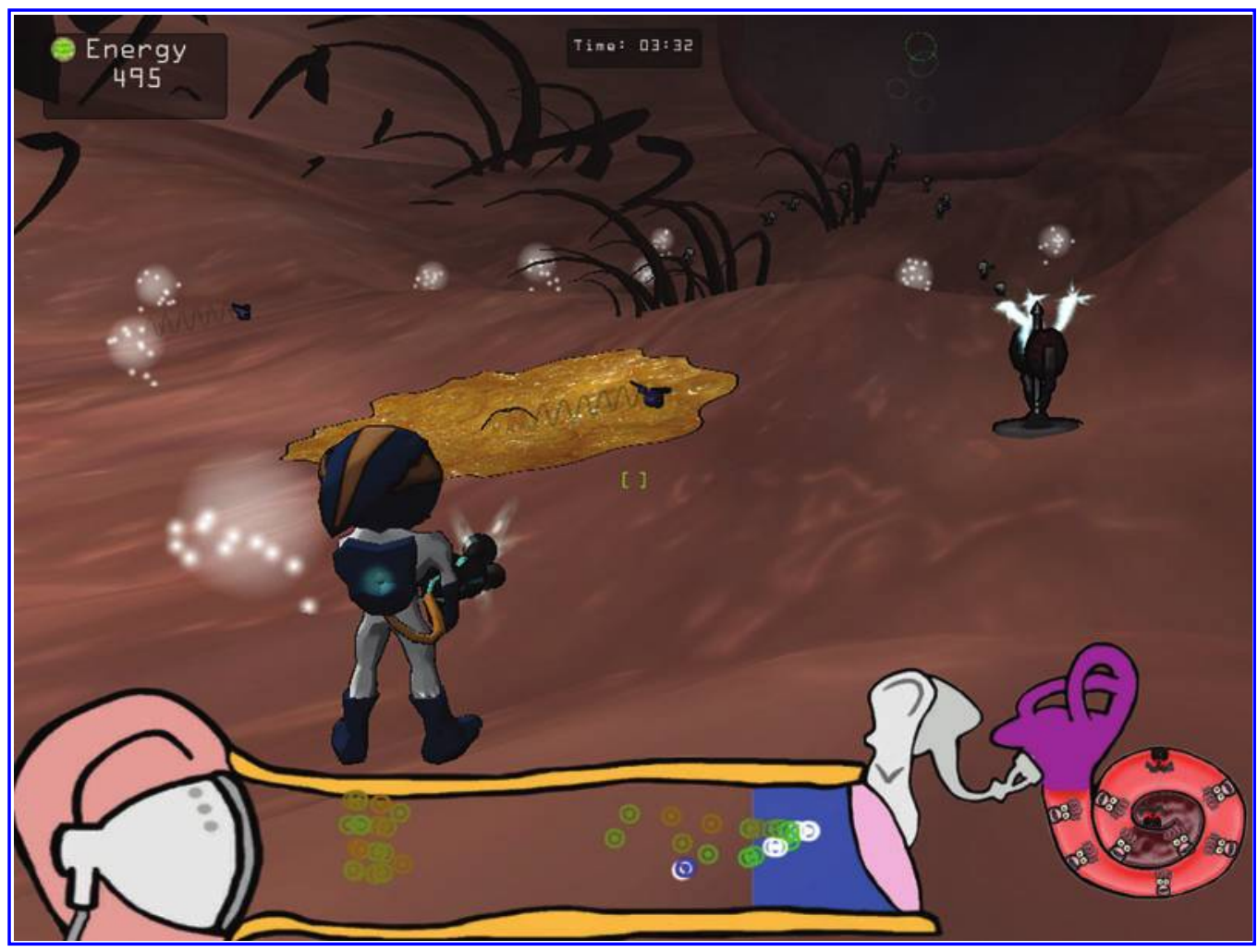

FIG. 2. "Epic Ear Defence" with two of the hair cell elements eliminated. An ear wax gland was been deployed to the floor of the canal, and a sapper tower is located at the right. A "tweeter" enemy is flying over the wax gland. The heads-up display also shows that the shield level is reduced, that some enemies are approaching the eardrum, and that another wave of enemies has recently entered the canal. Color images available online at www.liebertonline.com/g4h 
negotiate the development of a high-quality computer game. Conventional game methods and strategies as well as many elements of ear anatomy, the hearing system, and noiserelated hearing loss are all included in the game.

Further development of "Epic Ear Defence" is planned. Other game characters, including "Cheers for Ears Charlie," as well as more defense and enemy elements have been devised and will be implemented. Several difficulty levels and recording of high scores will also be made available. A micro-payment scheme for special game elements or levels is also being considered to cover some of the future development costs. Recognition of the game developers will also be added in the next version. Finally, implementation on mobile devices is also planned. This will require a review of the control of the game characters.

We are also commencing a thorough evaluation of the impact of the game on the players. A study is being planned to assess knowledge transfer related to hearing health and protecting hearing from dangerous sound levels and whether there is a change in listening behavior.

\section{Acknowledgments}

Chevron Australia provided funding through their Community Donations Scheme. Mr. Shri Rai, School of Information Technology, Murdoch University, provided mentoring for his students.

\section{Author Disclosure Statement}

No competing financial interests exist.

\section{References}

1. World Health Organization. The Global Burden of Disease: 2004 Update. Geneva: World Health Organization; 2008.

2. Daniel E. Noise and hearing loss: A review. J Sch Health 2007; 77:225-231.

3. Access Economics. Listen Hear! The Economic Impact and Cost of Hearing Loss in Australia. Canberra: Access Economics; 2006.

4. Niskar AS, Kieszak SM, Holmes AE, et al. Estimated prevalence of noise-induced hearing threshold shifts among children 6 to 19 years of age: The Third National Health and Nutrition Examination Survey, 1988-1994, United States. Pediatrics 2001; 108:40-43.

5. Kujawa SG, Liberman MC. Acceleration of age-related hearing loss by early noise exposure: Evidence of a misspent youth. J Neurosci 2006; 26:2115-2123.

Address correspondence to: Robert H. Eikelboom, BE, MApplSc, PhD Ear Science Institute Australia Suite 1, Level 2 1 Salvado Road Subiaco, WA 6009, Australia

E-mail: rob.eikelboom@earscience.org.au 